\title{
Molecular changes in bone marrow, tumor and serum after conductive ablation of murine 4T1 breast carcinoma
}

\author{
BEATA D. PRZYBYLA ${ }^{1}$, GAL SHAFIRSTEIN ${ }^{3}$, SAGAR J. VISHAL ${ }^{2}$, \\ RICHARD A. DENNIS ${ }^{4}$ and ROBERT J. GRIFFIN ${ }^{1}$
}

\author{
${ }^{1}$ Department of Radiation Oncology and ${ }^{2}$ College of Medicine, University of Arkansas for Medical Science, \\ Little Rock, AR; ${ }^{3}$ Department of Cell Stress Biology and Otolaryngology, Roswell Park Cancer Institute, Buffalo, NY; \\ ${ }^{4}$ Geriatric Research Education and Clinical Center, Central Arkansas Veteran Healthcare System, Little Rock, AR, USA
}

Received September 6, 2013; Accepted October 23, 2013

DOI: $10.3892 /$ ijo.2013.2185

\begin{abstract}
Thermal ablation of solid tumors using conductive interstitial thermal therapy (CITT) produces coagulative necrosis in the center of ablation. Local changes in homeostasis for surviving tumor and systemic changes in circulation and distant organs must be understood and monitored in order to prevent tumor re-growth and metastasis. The purpose of this study was to use a mouse carcinoma model to evaluate molecular changes in the bone marrow and surviving tumor after CITT treatment by quantification of transcripts associated with cancer progression and hyperthermia, serum cytokines, stress proteins and the marrow/tumor cross-talk regulator stromal-derived factor 1 . Analysis of 27 genes and 22 proteins with quantitative PCR, ELISA, immunoblotting and multiplex antibody assays revealed that the gene and protein expression in tissue and serum was significantly different between ablated and control mice. The transcripts of four genes (Cxcl12, Sele, $F g f 2$, Lifr) were significantly higher in the bone marrow of treated mice. Tumors surviving ablation showed significantly lower levels of the Lifr and Sele transcripts. Similarly, the majority of transcripts measured in tumors decreased with treatment. Surviving tumors also contained lower levels of SDF- $1 \alpha$ and HIF- $1 \alpha$ proteins whereas HSP27 and HSP70 were higher. Of 16 serum chemokines, IFN $\gamma$ and GM-CSF levels were lower with treatment. These results indicate that CITT ablation causes molecular changes which may slow cancer cell proliferation. However, inhibition of HSP27 may be necessary to control aggressiveness of surviving cancer stem cells. The
\end{abstract}

Correspondence to: Dr Beata D. Przybyla, Department of Radiation Oncology, University of Arkansas for Medical Sciences, Little Rock, 4301 West Markham Street, AR 72205, USA

E-mail: bea.przybyla@gmail.com

\section{Abbreviations: CITT, conductive interstitial thermal therapy}

Key words: thermal ablation, 4T1 tumors, bone marrow, gene expression, SDF1, HSP27, HSP70, Esele, Cxcl12, Fgf2, Lifr, cytokines changes in bone marrow are suggestive of possible increased recruitment of circulatory cancer cells. Therefore, the possibility of heightened bone metastasis after thermal ablation needs to be further investigated and inhibition strategies developed, if warranted.

\section{Introduction}

Thermal ablation therapy destroys cancer cells by delivering electromagnetic or acoustic energy [e.g. radiofrequency (1), laser (2), or focused ultrasound (3)] that is converted to heat in the target tissue. The therapy raises the temperature of the ablation site to between 56 and $100^{\circ} \mathrm{C}$ which results in irreversible damage by coagulation necrosis. Preclinical study with conductive interstitial thermal therapy (CITT) (4), (5) has demonstrated decreased metastasis in rabbits with VX2 tumors (6) and the reduction of hypoxia in tumor tissue surviving partial CITT ablation of murine breast carcinoma (7). Investigating the molecular changes associated with CITT and with other thermal ablation therapies may identify biomarkers that facilitate development of methods for monitoring and predicting the potential for therapy-associated enhancement of tumor re-growth, and metastasis.

Histological analysis of thermal ablation lesions in animal models has revealed three regions. The central region is adjacent to the ablation (i.e. area of probe insertion, or focus of ultrasound) and contains necrotic tumor tissue. Massive tumor debris appears to result in release of immunogenic factors (8). Adjacent to necrotic area is a coagulation margin that defines a sharp transition zone between necrotic and viable tissue. This highly hypoxic region was shown to be a site of accelerated tumor re-growth after radiofrequency ablation (9). A third region is more distant from the center of heat delivery and contains viable tumor tissue. In this region, we observed decreased hypoxia $72 \mathrm{~h}$ after CITT ablation (7). Thus, lesions of ablated tumor appear to possess areas with different properties that may have the potential to initiate induction of tumor re-growth (undesirable effect), stimulate antitumor immunity and decrease hypoxia (desirable effects).

Our research is interested in the mechanisms by which thermal tumor ablation affects communication between tumor tissue, bone marrow and sites of metastasis. Recent progress in 
understanding tumor biology has revealed crucial roles for the bone marrow in carcinogenesis, tumor growth and metastasis. Cytokines and growth factors secreted by tumor into circulation stimulate release of a variety of cells from bone marrow. These molecules contribute to tumor growth, vascularization, and regulation of immune cells in the tumor microenvironment $(10,11)$. However, little is known about the effects of thermal ablation upon the interactions between tumors, bone marrow, and site of metastasis. Thus, characterization of the molecules that potentially mediate these communications in the context of tumor growth and treatment would be an important step towards understanding the mechanisms underlying different outcomes of thermal tumor ablation.

The study presented here reports the preliminary results of quantitative analysis of gene expression and protein levels for serum, marrow and surviving tumor collected $72 \mathrm{~h}$ after partial ablation of $4 \mathrm{~T} 1$ carcinoma in $\mathrm{BALB} / \mathrm{c}$ mice. The CITT treatment protocol was designed to result in only partial tumor ablation to enable characterization of the remaining surviving tumor tissue. The study hypothesized that tumor ablation would broadly affect gene expression (Table I) and protein (Table II) levels for molecules involved in cell signaling, proliferation, adhesion, angiogenesis and stress response. Candidate molecules were chosen due to evidence in the literature for their role in breast tumor development and spreading, bone marrow homeostasis and tumor responses to thermal stress (12-16).

\section{Materials and methods}

Growing $4 T 1$ tumors in BALB/c mice and CITT treatment. The 4T1 mammary carcinoma is a syngeneic mouse model of breast cancer. The 4T1 cell line originated from spontaneously grown, metastatic mammary tumor in BALB/c mice. This mammary tumor has many characteristics of human invasive breast cancer (17). The thermal ablation procedures were described in detail in our previous study (7). The use of BALB/c mice (Jackson Laboratories, Bar Harbor, ME, USA) and the experimental protocol was approved by the University of Arkansas for Medical Sciences Institutional Animal Care and Use Committee. The 4T1 cancer cells $\left(2 \times 10^{5}\right)$ were injected subcutaneously into the right rear leg of each mouse. When tumor reached $10-12 \mathrm{~mm}$ in size, the mice were anesthetized with $1-2 \%$ isofluorane and a CITT probe, without pin deployment, was inserted in the center of a tumor. Temperatures were monitored with two thermocouples, one next to the tip of the CITT probe and another one, at the periphery of the tumor. Ablation was performed for $10 \mathrm{~min}$ with temperature maintained within the range $80-90^{\circ} \mathrm{C}$ (next to the CITT probe) while the peripheral tumor temperature remained in the low to mid $40^{\circ} \mathrm{C}$ range. Each mouse had one tumor. Five mice with tumors were ablated and four mice with tumors were used as untreated control.

Sample preparation. The mice were scarified $72 \mathrm{~h}$ after ablation. At this time point necrotic area is well established and the transition zone separating necrotic area from viable perinecrotic zone is clearly visible on tumor sections. The entire tumors were removed immediately after necropsy. One half of the tumor was used to prepare fresh sections in order to record the size of the ablated areas with a viability staining, triphenyltetrazolium chloride (TTC) as previously described (18). The other half of the tumor was used to prepare tissue samples from viable portion of tumors which were cut into pieces ranging from $50-100 \mathrm{mg}$, frozen in liquid $\mathrm{N}_{2}$ and stored at $-80^{\circ} \mathrm{C}$ until use. Femurs and tibiae were carefully cleaned from adherent soft tissue and placed in cold, sterile phosphate buffered saline, followed by transfer to Petri dish filled with Dulbecco's modified Eagle's medium (DMEM). The ends of bone were cut off with sterile scissors so that the bone marrow could be flushed with DMEM using a $3 \mathrm{cc}$ syringe and $27 \mathrm{G}$ needle. The cells suspensions were filtered through 70-mm BD Falcon nylon mesh (Fisher Scientific Inc., Sewanee, GA, USA). Cells were counted (Beckman Coutler Z series System, Hialeah, FL, USA), centrifuged at $1410 \mathrm{rpm}$ for $5 \mathrm{~min}$ at $4^{\circ} \mathrm{C}$, and re-suspended in serum free DMEM at a concentration $1 \times 10^{6}$ cells per $\mathrm{ml}$. Aliquots of $1.5 \mathrm{ml}$ of cells were made, centrifuged and the medium was removed so that cell pellets could be frozen in liquid $\mathrm{N}_{2}$ and stored at $-80^{\circ} \mathrm{C}$ until use.

Total RNA isolation and preparation of cDNA. Total RNA was isolated from frozen tumor and bone marrow cell pellets using the RNAqueous-4PCR kit (Ambion/Applied Biosystems, Foster City, CA, USA) according the manufacturer's instructions. RNA concentration was determined with ND-1000 Spectrophotometer (NanoDrop Technologies, Inc., Wilmington, DE, USA) and RNA integrity was verified with Bioanalyzer (Applied Biosystems). All RNA samples possessed an RNA integrity number $($ RIN) $>7$. cDNAs were prepared from $2 \mu \mathrm{g}$ of RNA using iScript cDNA Synthesis kit (Bio-Rad Laboratories, Inc., Hercules, CA, USA). This kit contains blend of oligo dT and random hexamers primers. The cDNAs were stored at $-20^{\circ} \mathrm{C}$ in $5-10 \mu \mathrm{l}$ aliquots. A pooled sample of all cDNAs was also prepared to use in primer testing and optimization of standard curves.

Quantititative RT-PCR. Primers used in the real-time polymerase chain reaction (RT-PCR) were designed using the murine NCBI nucleotides database and Primer Express v3.0 software (Table III). RT-PCR assays were performed according to the manufacturer's instructions using iTaqSYBR Green Supermix with ROX (Bio-Rad Laboratories Inc.), 384-well plates, and the ABI Prism 7900 Sequence Detection System (Applied Biosystems). For each transcript of interest standard curves were evaluated in order to optimize the primer concentration for maximum reaction efficiency. Standard curves were prepared from five-fold serial dilution of pooled cDNA. Gene expression was normalized to both $18 \mathrm{~S}$ ribosomal RNA and glyceraldehyde 3-phosphate dehydrogenase (GAPDH). The comparative $C_{T}\left(\Delta \Delta C_{T}\right)$ method for calculating relative gene expression was used to evaluate differences in levels of transcripts between control group (mice with non-treated tumors) and treated group (mice with ablated tumors) (19). The fold change of transcripts was calculated using DataAssist v3.0 software (Applied Biosystems).

Quantification of chemokines in serum. Blood was collected by cardiac puncture of anesthetized mice just before euthanasia. Isolated serum was aliquoted for storage in $-20^{\circ} \mathrm{C}$. Cytokines in serum were quantified with the Cytokine 
Table I. The genes analyzed in bone marrow and 4T1 tumors from BALB/c mice.

Symbol Molecule name Description (function in breast cancer)

Assay type: Quantitative RT-PCR in bone marrow and tumor lysates

Bmp2 Bone morphogenic protein 2

Ccl2 Chemokine (C-C motif) ligand 2 (MCP-1)

Csf3 Colony stimulating factor 3 (granulocyte) (G-CSF)

Csf3r Colony stimulating factor 3 receptor (granulocyte)

Cxcl12 Chemokine (C-X-C motif) ligand 12 (SDF-1)

Cxcr4 Chemokine (C-X-C motif) receptor 4 (SDF-1 receptor)

Fgf2 Fibroblast growth factor 2

Glg1 Golgi apparatus protein 1 (ESL-1)

Hsf1 Heat shock factor 1

Icam1 Intercellular adhesion molecule 1

Id1 Inhibitor of DNA binding 1

Il6 Interleukin 6

Il17ra Interleukin 17 receptor A

$K d r \quad$ Kinase insert domain protein receptor (VGFR2)

Klrk1 Killer cell lectin-like receptor subfamily number 1

Lif Leukemia inhibitory factor

Lifr Leukemia inhibitory factor receptor

Mmp2 Matrix metallopeptidase 2

Mmp9 Matrix metallopeptidase 9

Ptgs 2 Prostaglandin-endoperoxide synthase 2

Sele E-selectin

Serpin1 Serine protease inhibitor, nectin (PAI-1)

Tnfrsflla Tumor necrosis factor receptor superfamily, Expressed on osteoclasts and dendritic cells - bone metastasis member 11a (RANK)

Tnfsf11 Tumor necrosis factor (ligand) superfamily, member 11 (RANKL)

Tnf Tumor necrosis factor

Ucp2 Uncoupling protein 2 (mitochondrial proton carrier)

Vcam1 Vascular cell adhesion molecule 1
Growth factor, TGF $\beta$ superfamily - tumor apoptosis, EMT

Signaling molecule - TAM infiltration, cancer stem cell

Cytokine-apoptosis, neutrophils release from BM

CD114, hematopoietin receptor family - cell maturation in BM

Signaling molecule - hematopoietic cell homing and quiescence, communication between tumor and BM

CD184 (fusin), $\alpha$-chemokine receptor - metastasis to BM

Growth factor (basicFGF) - myelopoiesis and angiogenesis in BM

Glycoprotein, leukocyte ligand for E-selectin-metastasis

Nuclear protein activating HSPs-growth, inhibition of apoptosis

CD54, glycoprotein, immunoglobulin family-metastasis

Nuclear protein - metastasis, inhibition of differentiation

Pleiotropic cytokine - metastasis to BM

CD217, glycoprotein - proinflamatory, associated with poor prognosis

VGEF receptor - angiogenesis, tumor progression

CD314 (NKG2D), NK cell receptor-antitumor immunity

Pleiotropic cytokine-tumor proliferation, chemoattractant for BM derived cells

CD118, cytokine receptor - suppressor of metastasis

Type IV collagenase, gelatinase A - metastasis

Type IV collagenase, gelatinase A - angiogenesis, metastasis

Cyclooxygenase 2 (COX2) enzyme - worse prognosis

CD62, adhesion molecule, expressed only on activated endothelial cells - metastasis

Inhibitor of tissue plasminogen activator (tPA) - cancer progression

CD254, expressed on osteoblasts, stromal and T cells - cancer cells chemoattractant

Cachectin, TNF $\alpha$-cytotoxin - breast cancer promoter

Mitochondrial ROS regulator - tumor promoting factor

CD106, mediator of immune cell adhesion to endothelium - aberrantly expressed on breast cancer cells, metastasis 16-plex panel (Quansys Biosciences, Inc., Logan, UT, USA). This chemiluminescent Quansys Q-plex Array contained IL-1 $\alpha$, IL-1 $\beta$, IL-2, IL-3, IL-4, IL-5, IL-6, IL-10, IL-12, IL-17, MCP-1, IFN $\gamma$, TNF $\alpha$, MIP-1 $\alpha$, GM-CSF and RANTES antibodies absorbed to each well of a 96-well plate (Table II). The lower level of detection was different for each cytokine and ranged from $0.1 \mathrm{pg} / \mathrm{ml}(\mathrm{TNF} \alpha)$ to $5.0 \mathrm{pg} / \mathrm{ml}$ (MCP-1). Serum samples were tested in triplicate. Data were collected 
Table II. List of proteins analyzed in 4T1 tumors and serum from BALB/c mice.

$\begin{array}{lll}\text { Symbol } & \text { Molecule name } \quad \text { Description (function in breast cancer) }\end{array}$

Assay type: ELISA in tumor lysates

CXCL12 Chemokine (C-X-C motif) ligand 12

(SDF-1)

Assay type: Western immunoblotting in tumor lysates

HIF-1 $\alpha$ Hypoxia-inducible factor 1A

HSP27 Heat shock protein 27

HSP70 Heat shock protein 70

MMP9 Matrix metallopeptidase 9

Ki67 Antigen Ki-67

Assay type: Antibodies multiplex of 16 murine chemokines in serum

IL-1 $\alpha$, IL-1 $\beta$, IL-2, IL-3, IL-4, IL-5, IL-6, IL-10,

IL-12, Il-17, MCP-1, IFN $\gamma$, TNF $\alpha$, MIP-1 $\alpha$, GM-CSF, RANTES
Signaling molecule - hematopoietic cell homing and quiescence, communication between tumor and BM

Transcription factor - adaptation to hypoxia, overexpression in invasive cancers

Stress response protein - drug resistance, stem cell maintenance

Stress response protein - antigen binding, anti-apoptotic

Type IV collagenase, gelatinase A - angiogenesis, metastasis

Nuclear protein - marker of proliferation

Signaling molecules with immunomodulatory and chemotaxic functions - altered in serum of breast cancer patients

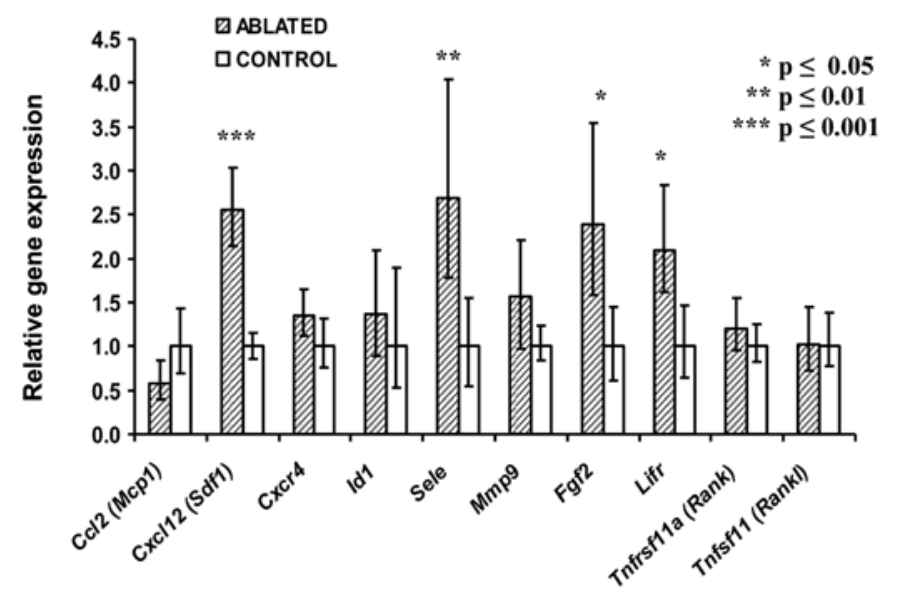

Figure 1. Transcript levels in RNA from bone marrow of BALB/c mice. Quantitative real-time RT-PCR was used to quantify 27 genes of interest in marrow isolated from treated $(\mathrm{N}=5)$ and control $(\mathrm{N}=4)$ mice $72 \mathrm{~h}$ after partial thermal ablation of 4T1 mammary carcinoma, subcutaneously grown in BALB/c mice. Expression data for the genes of interest was normalized to $18 \mathrm{~S}$ levels. Bars represent the relative expression (RQ) compared between marrows from ablated versus control mice. RQ is calculated as $2^{-(\Delta \Delta C T)}$ and error bars represent RQ $\min =2^{-(\Delta \Delta C T+S T D)}$ and RQ $\max =2^{-(\Delta \Delta C T-S T D)}$. Results are shown for 10 of the 27 transcripts quantified by the study.

using Q-View Imager and software (Quansys Biosciences Inc.). Results are presented in picograms of cytokine per $\mathrm{ml}$ of serum $(\mathrm{pg} / \mathrm{ml})$.

ELISA. Tumor lysates were prepared from frozen tumors by homogenization in diluted Cell Lysis Buffer 10X (Cell Signaling Technology, Inc., Beverly, MA, USA) containing $1 \mathrm{mM}$ PMSF (phenylmethanesulfonyl fluoride). The total protein concentration of lysates was determined with Pierce BCA Protein Assay kit, (Thermo Fisher Scientific Inc., Rockford, IL, USA), a Synergy HT spectrophotometer and the Gene5 software (BioTech Instruments, Winooski, VT, USA). The level of stromal derived factor 1 (SDF-1, also known as chemokine CXCL12) in the lysates was determined using Duo Set ELISA Development kit, according the manufacturer's instructions (cat \# DY460, R\&D Systems, Inc., Minneapolis, MN, USA). Samples were assessed in triplicate. The results are presented as picograms of SDF-1 protein per micrograms of total protein content of the tumor lysate.

Western blotting. Tumor lysates for immunoblotting were prepared as described above for ELISA. Novex Pre-Cast gradient gels (4-20\%) and NuPAGE Electrophoresis system (Invitrogen Life Technologies, Inc., Carlsbad, CA, USA) were used to resolve proteins from $8 \mu \mathrm{g}$ of total protein sample per well. Western transfer onto polyvinylidene difluoride membranes (Amersham, Piscataway, NJ, USA) was performed in an XCell II Blot Module (Invitrogen Life Technologies, Inc.). After protein transfer, membranes were stored at $4^{\circ} \mathrm{C}$. For immunoblotting, membranes were incubated in $10 \%$ fat-free powdered milk solution for $4 \mathrm{~h}$ at room temperature (RT). Primary antibodies were applied for $1 \mathrm{~h}$ at RT, the membrane was washed, and the secondary antibody was applied for $1 \mathrm{~h}$ at RT. The following primary antibodies were used: monoclonal anti-HIF-1 $\alpha$ (cat \# NB100-105, Novus Biologicals), polyclonal anti-HSP 27 (cat \# sc-1049, Santa Cruz Biotechnology), monoclonal anti-HSP 70 (cat \# sc-24, Santa Cruz Biotechnology), monoclonal anti-Ki67 (cat \# NBP140684, Novus Biologicals) rabbit polyclonal anti-MMP9 (cat \# ab38898, Abcam) and anti-actin (1-19) (cat \# sc1616, Santa Cruz Biotechnology). The secondary antibodies were: 
Table III. The murine primers used.

\begin{tabular}{|c|c|c|c|}
\hline Gene & Accession no. & Forward primer & Reverse primer \\
\hline Bmp2 & NM_007553 & TGTCCCCAGTGACGAGTTTCT & CCTGTATCTGTTCCCGGAAGAT \\
\hline Ccl2 & NM_011333 & CTGAAGCCAGCTCTCTCTTCCT & CAGGCCCAGAAGCATGACA \\
\hline$C s f 3$ & NM_009971 & CAGTACCCCCAAAAAATCAGTGA & TGGGCCCCCCTGAGAT \\
\hline$C s f 3 r$ & NM_007782 & TCCAGCGAGTCCCCAAAG & CAGCATGGGAGGCTCCAAT \\
\hline Cxcl12 & NM_021704 & GCCTCCAAACGCATGCTT & ATTGGTCCGTCAGGCTACAGA \\
\hline Cxcr4 & NM_009911 & TCGGCAATGGATTGGTGAT & CCGTCATGCTCCTTAGCTTCTT \\
\hline$F g f 2$ & NM_008006 & TGGTATGTGGCACTGAAACGA & TCCAGGTCCCGTTTTGGAT \\
\hline Glg1 & NM_009149 & CTCACTGCGCCCTCTAACG & GGCACCTGATGCTGCTCTACT \\
\hline Hsfl & NM_008296 & CATAAAAATACGCCAGGACAGTGT & CCСCTTCATCAGCTGCACAT \\
\hline Icaml & NM_010493 & TGGCGGGAAAGTTCCTGTT & TCCAGCCGAGGACCATACA \\
\hline Idl & NM_010495 & GAACGTCCTGCTCTACGACATG & TGGGCACCAGCTCCTTGA \\
\hline Il-17ra & NM_008359 & CCCAGGCAAGAAGAATTCCA & CACCAGTGAAACTTGCTTAGAGTGA \\
\hline Il-6 & NM_031168 & CCACGGCCTTCCCTACTTC & TTGGGAGTGGTATCCTCTGTGA \\
\hline$K d r$ & NM_010612 & ACTGCAGTGATTGCCATGTTCT & TCATTGGCCCGCTTAACG \\
\hline Klrk1 & NM_033078 & GGCAATTCGATTCACCCTTAAC & ATACTGGCTGAAACGTCTCTTTGA \\
\hline Lif & NM_008501 & GCCACGGCAACCTCATG & ATTGGCGCTGCCATTGA \\
\hline Lifr & NM_013584 & AGAACATCACTGACATATCCCAGAAG & GTATAGGCTCGCAGGACCAGAT \\
\hline Mmp2 & NM_008610 & GGACCCCGGTTTCCCTAA & CAGGTTATCAGGGATGGCATTC \\
\hline Mmp9 & NM_013599 & TGGTGGCAGCGCACG & CTTCCGGCACGCTGGA \\
\hline Ptgs 2 & NM_011198 & TGCCTCCCACTCCAGACTAGA & CAGCTCAGTTGAACGCCTTTT \\
\hline Sele & NM_011345 & TCCTGCGAAGAAGGATTTGAA & CCСCTCTTGGACCACACTGA \\
\hline Serpin 1 & NM_008871 & CCGTGGAACAAGAATGAGATCAG & CTCTAGGTCCCGCTGGACAA \\
\hline$T g f b 1$ & NM_011577 & GCAGTGGCTGAACCAAGGA & AGCAGTGAGCGCTGAATCG \\
\hline $\operatorname{Tnf}$ & NM_013693 & CACAAGATGCTGGGACAGTGA & TCCTTGATGGTGGTGCATGA \\
\hline Tnfrsfl1a & NM_009399 & TCGTCCACAGACAAATGCAAA & GTGTGCTTCTAGCTTTCCAAGGA \\
\hline Tnfsfl1 & NM_011613 & GGCCACAGCGCTTCTCA & CCTCGCTGGGCCACATC \\
\hline Ucp 2 & NM_011671 & GCCCCTTCACCTCTTTAGCA & CCAAGCACTGGGAAGGTCTAAC \\
\hline Vcaml & NM_011693 & СТCССCTGAATACAAAACGATTG & GCCCGTAGTGCTGCAAGTG \\
\hline
\end{tabular}

anti-mouse (cat \# 170-6516; Bio-Rad Laboratories, Inc.), anti-goat (cat \# sc-2020; Santa Cruz Biotechnology) and antirabbit (cat \# sc-2004, Santa Cruz Biotechnology).

Statistical analysis. The two-tailed Student's t-test was used to analyze RT-PCR and ELISA data. Mann-Whitney U test and Fisher's exact test were used to evaluate cytokines panel results. The software DataAssist v3.0 (Applied Biosystems) and Sigma Plot v11 (Systat Software Inc., San Jose, CA, USA) were applied.

\section{Results}

Effects on bone marrow gene expression. The effects on the bone marrow of BALB/c mice $72 \mathrm{~h}$ after tumor ablation by CITT therapy were measured by quantitative real-time RT-PCR. Marrow RNA was assayed for 27 murine transcripts (Table I). Four transcripts were expressed at significantly higher levels for the mice treated with tumor ablation $(\mathrm{N}=5)$ as compared to the control mice $(\mathrm{N}=4)$ with untreated tumors (Fig. 1). Transcript levels for genes encoding stromal derived factor 1 (Cxcl12), E-selectin (Sele), leukemia inhibitory factor 1 receptor ( Lifr $)$ and basic fibroblast growth factor $(F g f 2)$ ranged from an average of 2 to 2.5 -fold higher $(\mathrm{P}<0.05)$ in the treated as compared to the untreated mice. Transcripts of other genes such as chemokine ligand-2 (Ccl2) and chemokine receptor-4 (Cxcr4) were not affected in bone marrow by tumor ablation (Fig. 1). Four other examples of transcripts unaffected by the treatment are also shown.

Effects on surviving tumor gene expression. The effects on viable 4T1 tumor surrounding the site of partial ablation were also measured by quantitative real-time RT-PCR. Tumor RNA was assayed for 27 murine transcripts (Table I) for treated $(\mathrm{N}=5)$ and control mice $(\mathrm{N}=4)$. Two transcripts were expressed at significantly lower levels for the mice treated with tumor ablation $(\mathrm{N}=5)$ as compared to the control mice $(\mathrm{N}=4)$ with untreated tumors (Fig. 2). Transcript levels of Lifr and Sele genes for treated tumors was only $\sim 60 \%$ as high as the control tumors $(\mathrm{P}<0.05)$. Transcripts of other genes such as matrix metallopeptidase-9, serpin-1 and vascular cell adhesion molecule-1 were not affected in tumor tissue that survived ablation (Fig. 2).

Effects on surviving tumor protein level. The effects of partial tumor ablation on protein levels in viable tissue 


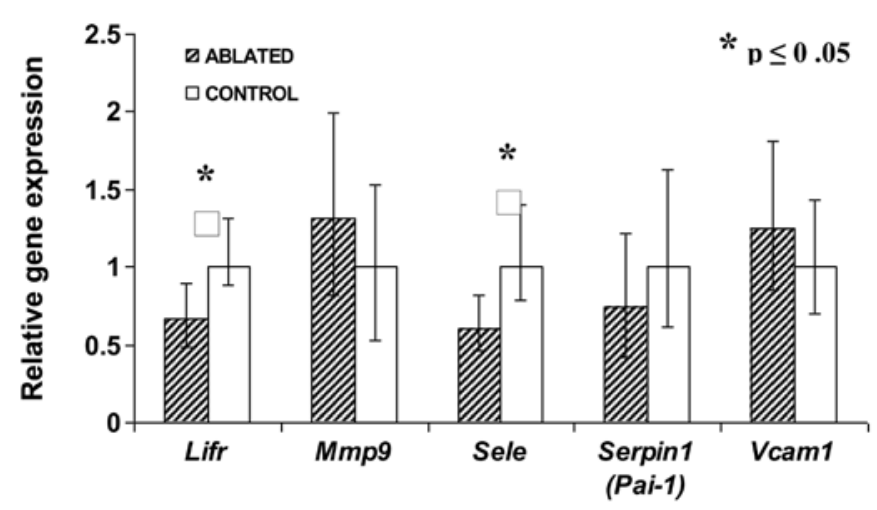

Figure 2. Transcript levels in RNA from viable 4T1 tumors. Quantitative real-time RT-PCR was used to quantify 27 genes of interest in RNA isolated from viable tumor of treated $(\mathrm{N}=5)$ and control $(\mathrm{N}=4)$ mice $72 \mathrm{~h}$ after partial thermal ablation. Expression data for the genes of interest were normalized to $18 \mathrm{~S}$ levels. Bars represent the relative expression (RQ) compared between marrows from ablated versus control mice. RQ is calculated as $2^{-(\Delta \Delta C T)}$ and

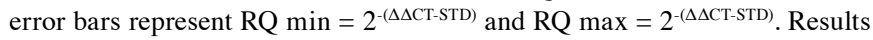
are shown for 5 of the 27 transcripts quantified by the study.

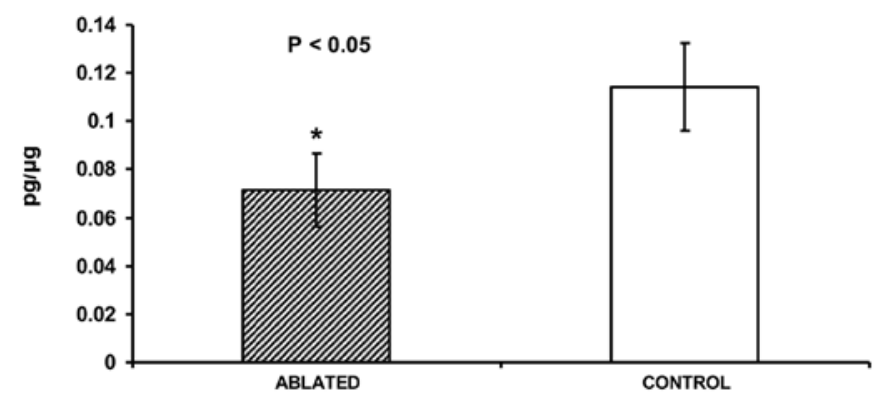

Figure 3. Protein levels for SDF1 in 4T1 tumor lysates. ELISA immunoassay was used to measure SDF1 protein levels in lysates of viable tumor from treated $(\mathrm{N}=4)$ and control $(\mathrm{N}=3)$ mice $72 \mathrm{~h}$ after partial thermal ablation. Data are expressed in pg per $\mu \mathrm{g}$ of total protein and are presented as mean values \pm standard deviation.

around the site of ablation $72 \mathrm{~h}$ after treatment were measured by immunoassays. As measured by ELISA, levels of SDF-1 protein in tumor lysates were significantly lower $(0.071 \pm 0.016 \mathrm{pg} / \mu \mathrm{g}$ versus $0.113 \pm 0.017 \mathrm{pg} / \mu \mathrm{g}, \mathrm{P}<0.05)$ for ablated $(\mathrm{N}=4)$ as compared to control $(\mathrm{N}=3)$ tumor tissue (Fig. 3). Levels of six proteins were measured in tumor lysates using western immunoblotting (Table II). A representative blot showing results for these proteins and actin, a control protein, are presented (Fig. 4). Levels of heat shock proteins HSP70 and HSP27 were higher for all individual samples for ablated $(\mathrm{N}=5)$ as compared to control $(\mathrm{N}=4)$ tumor tissue; whereas levels of hypoxia inducible factor (HIF-1 $\alpha$ ) were consistently lower for ablated tumors. Protein levels for matrix metallopeptidase- 9 and antigen Ki- 67 were unaffected by the treatment (data not shown).

Effects on serum cytokine levels. The effects of partial tumor ablation on serum cytokine levels $72 \mathrm{~h}$ after treatment were measured by multiplex immunoassay. The results for 10 of the 16 cytokines are presented (Fig. 5). The cytokines IL-1 $\alpha$, IL-17, IFN $\gamma$, TNF $\alpha$ and GM-CSF were present in serum at low concentrations. Other cytokines including IL-1 $\beta$, IL-5, IL-6,

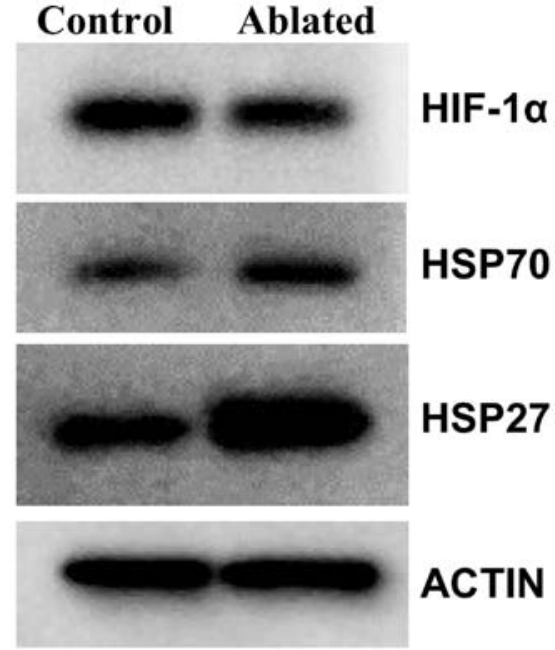

Figure 4. Protein levels for HIF1 $\alpha$ and heat shock proteins in 4T1 tumor lysates. Western immunoblotting was used to measure protein levels of HIF1 $\alpha$, Hsp70 and Hsp27 in lysates of a viable tumor isolated from treated $(\mathrm{N}=5)$ and control $(\mathrm{N}=4)$ mice $72 \mathrm{~h}$ after partial thermal ablation. A representative blot is shown. Actin was used as the control protein.

MCP-1 and RANTES were present at higher concentrations. These ten cytokines were present at detectable levels in serum from at least five of the nine study mice and the inter-sample variability within groups was large. Only two cytokines, interferon- $\gamma$ (IFN $\gamma$ ) and granulocyte-macrophage colonystimulating factor (GM-CSF) showed significant differences between groups. IFN $\gamma$ and GM-CSF had a significantly lower level in serum from the ablated mice as compared to control mice $(\mathrm{P}<0.05)$. Notably, interleukin $1 \beta$ showed a trend towards lower values $(\mathrm{P}=0.154)$ in serum from ablated mice.

\section{Discussion}

Thermal ablation has been used to treat tumors of the liver (20), prostate (21), and breast (22-24) in humans and animal models . Results of these therapies can vary greatly depending on many variables, including ablation modality, operator techniques and tumor site. For example, radiofrequency ablation can lead to accelerated perinecrotic outgrowth of colorectal liver metastases (25); whereas, we previously demonstrated that CITT therapy decreased metastasis in rabbits with VX2 tumors (6). In the current study, a mouse mammary carcinoma model was used to characterize the molecular changes that occur in serum, bone marrow, and tumor surviving partial ablation. At the transcriptional level, ablation increased Sele, Fgf2, Lifr and Cxcl12 in marrow and decreased Lifr and Sele in the surviving tumor. At the protein level, ablation resulted in decreased levels of SDF1 and HIF-1 $\alpha$ and increased levels of HSP27 and HSP70 in the surviving tumor. In serum, ablation decreased the concentration of the IFN $\gamma$ and GM-CSF. Thus, 10 molecules have been identified that may be involved with alteration of communication between the marrow and tumor as well as alteration of homeostasis in surviving tumors.

The bone marrow responded to thermal ablation with an increase in the Sele, Fgf2, Lifr and Cxcl12 transcripts. Sele encodes E-selectin, an adhesion molecule expressed by activated endothelial cells while tumor cells express E-selectin 

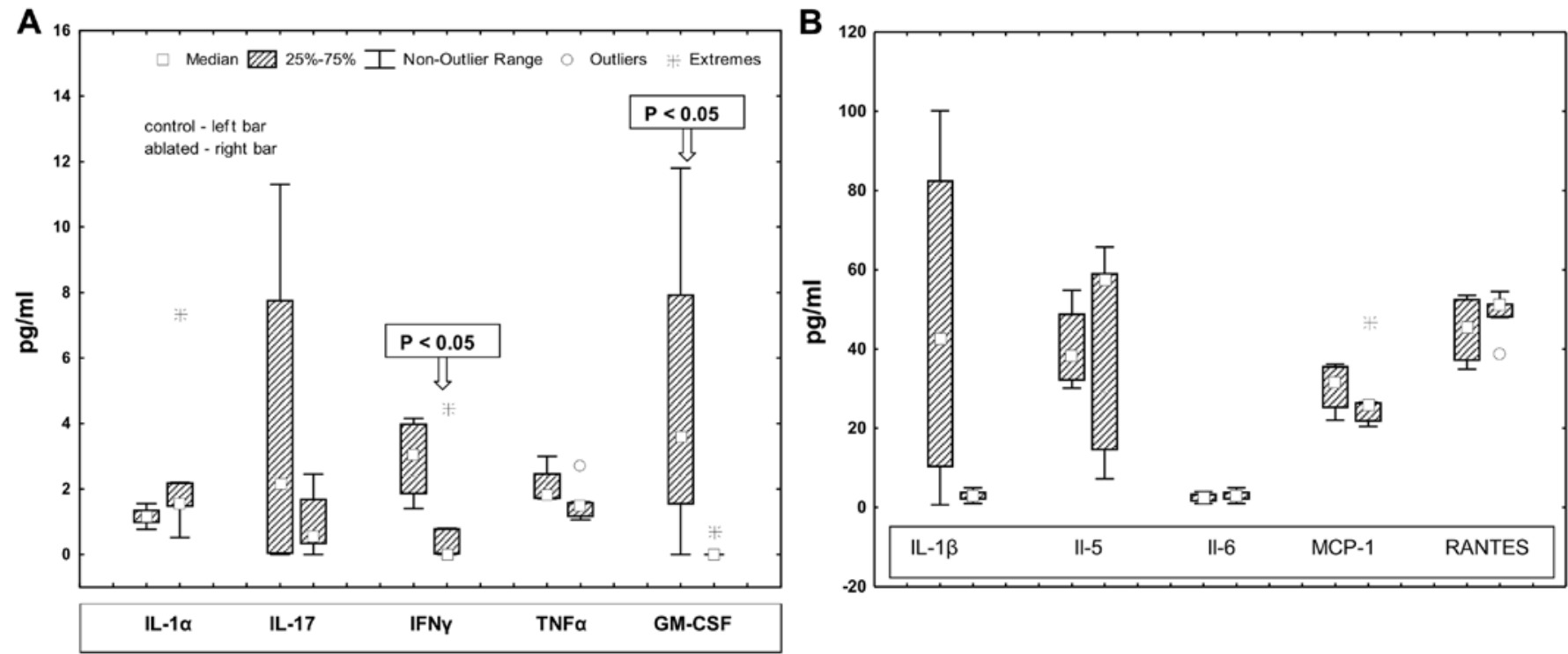

Figure 5. Cytokine levels in serum. Quansys multiplex immunoassay was used to measure 16 cytokines in serum isolated from treated (N=5) and control $(\mathrm{N}=4)$ mice $72 \mathrm{~h}$ after partial thermal ablation. Of the 16,10 were present at detectable levels. (A) Cytokines present at up to $15 \mathrm{pg} / \mathrm{ml}$. (B) Cytokines present at up to $100 \mathrm{pg} / \mathrm{ml}$.

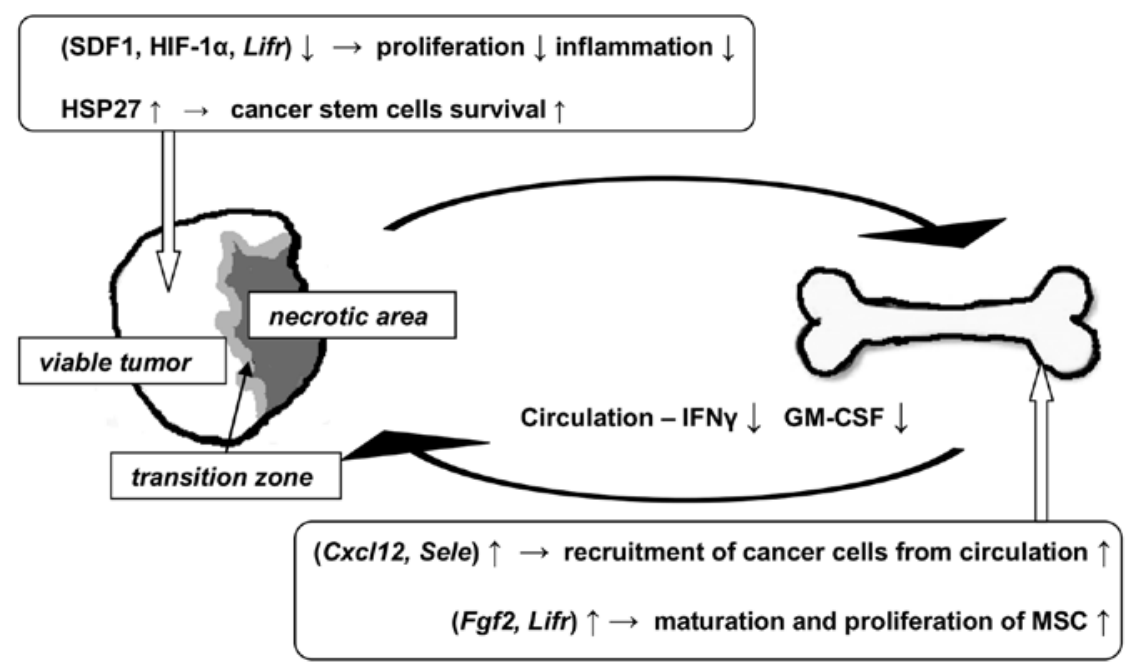

Figure 6. Summary results and hypothetical effects of molecular responses to tumor ablation. The molecular changes identified in this study after thermal ablation of mouse mammary carcinoma suggest decreased proliferation for surviving tumor cells and decreased local and systemic inflammation. These changes are accompanied by molecular evidence for increased maturation and proliferation of bone marrow cells and recruitment of circulatory cancer cells to the marrow. Elevated HSP27 in the perinecrotic area may be a marker of a robust population of cancer stem cells that have survived tumor ablation.

ligands. Interactions between these cell types through E-selectin are thought to regulate cancer metastases (26). Thus, the possibility exists that increased E-selectin could promote adhesion of cancer cells and enhance seeding tumor cells into the marrow. Fibroblasts growth factor 2 is a stromal cell mitogen and stimulates myelopoiesis in marrow. However, in the presence of advanced, untreated human breast and lung tumors, low levels of Fgf2 result in an arrest of maturation of mesenchymal stromal cells in marrow (27). The response of $F g f 2$ to ablation seen here may be an attempt of the marrow to re-establish normal maturation for mesenchymal cells.

Leukemia inhibitory factor (LIF) is a cytokine that also affects marrow mesenchymal cells $(28,29)$. Upregulation of the LIF receptor transcript (Lifr) in marrow may indicate that tumor ablation influences not only maturation but also differentiation of mesenchymal stromal cells. Stromal derived factor 1 is a signaling molecule for communication between tumor and marrow $(30,31)$. The SDF1 receptor (CXCR4) is expressed on breast and other epithelial cancer cells and has been shown in multiple studies to be involved with metastasis (32-35). Increase of expression of the Cxcl12 transcripts encoding SDF1 in bone marrow may stimulate mobilization and recruitement of immature hematopoietic cells, endothelial and smooth muscle progenitor to neo-angiogenic niche $(36,37)$. However, it is unknown whether or not the elevation of Cxcl12 transcript found in marrow post-ablation is evidence of seeding premetastatic niches or the primary tumor site. In 
summary, four transcripts have been identified which respond in marrow to tumor ablation. Further investigation is needed to confirm whether or not their encoded proteins play a role in mesenchymal cells development or tumor dissemination after thermal ablation.

Tumor tissue responded to thermal ablation with a decrease in SDF1 and HIF-1 $\alpha$ proteins, and an increase in HSP27 and HSP70 proteins, and decreases in Lifr and Sele genes transcripts. Our work previously showed that thermal ablation decreases hypoxia in surviving tumor areas (7). The results presented here are consistent with this effect in that SDF1 and hypoxia inducible factor $1(\mathrm{HIF}-1 \alpha)$ are lower in treated than untreated tumors. These changes could inhibit the metastatic potential of surviving tumor cells or inhibit signaling between the marrow and tumor for the recruitment of cells supportive of tumor growth such as pro-angiogenic endothelial progenitor cells or marrow suppressor cells $(32,35,38,39)$. The increase in heat shock proteins -70 and -27 after treatment may be a stress response. Unfortunately, HSP70 may be acting to protect the cancer cells as its expression has been associated with poor prognosis (40). However, HSP70 may also be indicative of a boost in antitumor immune activity post-ablation (41). Heightened tumor HSP27 is also associated with poor prognosis and metastasis (42) though evidence indicates multiple potential roles in tumorigenesis including promotion of tumor growth (43), regulation of epithelial-mesenchymal transition (44), and conferral of chemo-resistance (45). The increase of HSP27 implies that this heat shock protein should be closely monitored during thermal ablation of tumors and may be a useful marker to assist in determining appropriate treatment regimens in combination with thermal ablation. The effects of ablation on the Sele and Lifr genes were the opposite in tumor to that of marrow where we proposed that these factors may be involved with metastasis and mesenchymal cell development. It would be beneficial if the decrease seen in tumor was the result of a dampened potential for tumor cell proliferation and metastasis post-treatment. In summary, two transcripts and four proteins have been identified which respond in tumor tissue to ablation though additional pre-clinical studies are needed to define their functions and understand whether these molecules are involved with pro- or anti-tumorigenic responses after thermal ablation.

Serum analysis revealed that thermal ablation decreased concentrations of the granulocyte-macrophage stimulating factor (GM-CSF) and interferon- $\gamma($ IFN $\gamma)$. These molecules function in inflammation and the immune response. Their levels are influenced both by the presence of solid tumors $(46,47)$ and thermal ablation (48). However, the significance of their low levels post-ablation is unknown. These cytokines may be indicative of decreased need for antitumor immune function, immunosuppression by the treatment, or they may be associated with slowed tumor growth or smaller number of tumor cells after partial ablation $(49,50)$. Future studies will be needed to determine the source, effects and meaning of low systemic levels of these cytokines three days after ablation therapy.

The major findings and proposed implications of the study are summarized in Fig. 6. Thermal ablation appears to decrease local and systemic decrease of inflammation. Proliferation of viable tumor cells may decrease but HSP27 may indicate the presence of a protected population of surviving cancer cells that may be responsible for aggressive tumor re-growth. Additionally, thermal ablation caused upregulation of genes in bone marrow that are associated with maturation and proliferation of mesenchymal stem and hematopoietic cells to possibly replenish immune cells used during the initial response to trauma. The increase of gene expression encoding SDF1, a ligand for cancer cells, could increase the risk for circulating cancer cells to establish themselves in marrow. We conclude that thermal ablation clearly impacts transcript and protein levels of molecules that may be involved in pro- and anti-tumor activity in the tumor itself, in bone marrow and in serum. However, additional studies are needed to determine the influence of these changes on cell phenotype and their significance relative to cancer host or patient outcomes.

\section{Acknowledgements}

We thank Jessica S. Webber for excellence in performing animal experiments and Azemat Jamshidi-Parsian for help in preparation of western immunoblotting assay. This work was supported, in part, by a grant from Fashion Footwear Association of New York (FFANY/QVC) to G.S. and R.J.G. and by NIH grants CA44114 (R.J.G).

\section{References}

1. Shah DR, Green S, Elliot A, McGahan JP and Khatri VP: Current oncologic applications of radiofrequency ablation therapies. World J Gastrointest Oncol 5: 71-80, 2013.

2. Oto A, Sethi I, Karczmar G, et al: MR imaging-guided focal laser ablation for prostate cancer: phase I trial. Radiology 267: 932-940, 2013.

3. Merckel LG, Bartels LW, Kohler MO, et al: MR-guided highintensity focused ultrasound ablation of breast cancer with a dedicated breast platform. Cardiovasc Intervent Radiol 36: 292-301, 2013.

4. Shafirstein G, Hennings L, Kaufmann Y, et al: Conductive interstitial thermal therapy (CITT) device evaluation in VX2 rabbit model. Technol Cancer Res Treat 6: 235-246, 2007.

5. Shafirstein G, Novák P, Moros EG, et al: Conductive interstitial thermal therapy device for surgical margin ablation: in vivo verification of a theoretical model. Int J Hyperthermia 23: 477-492, 2007.

6. Shafirstein G, Kaufmann Y, Hennings L, et al: Conductive interstitial thermal therapy (CITT) inhibits recurrence and metastasis in rabbit VX2 carcinoma model. Int J Hyperthermia 25: 446-454, 2009.

7. Przybyla BD, Shafirstein G, Koonce NA, Webber JS and Griffin RJ: Conductive thermal ablation of 4T1 murine breast carcinoma reduces severe hypoxia in surviving tumour. Int $\mathbf{J}$ Hyperthermia 28: 156-162, 2012

8. Dromi SA, Walsh MP, Herby S, et al: Radiofrequency ablation induces antigen-presenting cell infiltration and amplification of weak tumor-induced immunity. Radiology 251: 58-66, 2009.

9. van der Bilt JD, Soeters ME, Duyverman AM, et al: Perinecrotic hypoxia contributes to ischemia/reperfusion-accelerated outgrowth of colorectal micrometastases. Am J Pathol 170: 1379-1388, 2007.

10. Peters BA, Diaz LA, Polyak K, et al: Contribution of bone marrow-derived endothelial cells to human tumor vasculature. Nat Med 11: 261-262, 2005.

11. Bunt SK, Sinha P, Clements VK, Leips J and OstrandRosenberg S: Inflammation induces myeloid-derived suppressor cells that facilitate tumor progression. J Immunol 176: 284-290, 2006.

12. Di Rosa F: T-lymphocyte interaction with stromal, bone and hematopoietic cells in the bone marrow. Immunol Cell Biol 87: 20-29, 2009.

13. Evans SS, Fisher DT, Skitzki JJ and Chen Q: Targeted regulation of a lymphocyte-endothelial-interleukin- 6 axis by thermal stress. Int J Hyperthermia 24: 67-78, 2008. 
14. Peer AJ, Grimm MJ, Zynda ER and Repasky EA: Diverse immune mechanisms may contribute to the survival benefit seen in cancer patients receiving hyperthermia. Immunol Res 46: $137-154,2010$.

15. Baronzio G, Gramaglia A and Fiorentini G: Hyperthermia and immunity. A brief overview. In Vivo 20: 689-695, 2006.

16. Chantrain CF, Feron O, Marbaix E and DeClerck YA: Bone mrrow microenvironment and tumor progression. Cancer Microenviron 1: 23-35, 2008

17. Heppner GH, Miller FR and Shekhar PM: Nontransgenic models of breast cancer. Breast Cancer Res 2: 331-334, 2000.

18. Lippold HJ: Quantitative succinic dehydrogenases histochemistry. A comparison of different tetrazolium salts. Histochemistry 76 381-405, 1982.

19. Schmittgen TD, Lee EJ and Jiang J: High-throughput real-time PCR. Methods Mol Biol 429: 89-98, 2008.

20. Wiggermann P, Zeman F, Niessen C, et al: Percutaneous irreversible electroporation (IRE) of hepatic malignant tumours: contrast-enhanced ultrasound (CEUS) findings. Clin Hemorheol Microcirc 52: 417-427, 2012.

21. Uchida T, Nakano M, Hongo S, et al: High-intensity focused ultrasound therapy for prostate cancer. Int J Urol 19: 187-201, 2011.

22. Zhao Z and Wu F: Minimally-invasive thermal ablation of earlystage breast cancer: a systemic review. Eur J Surg Oncol 36: $1149-1155,2010$

23. Yoon J, Cho J, Kim N, et al: High-frequency microwave ablation method for enhanced cancer treatment with minimized collateral damage. Int J Cancer 129: 1970-1978, 2011.

24. Hung WK, Mak KL, Ying M and Chan M: Radiofrequency ablation of breast cancer: a comparative study of two needle designs. Breast Cancer 18: 124-128, 2011.

25. Nijkamp MW, van der Bilt JD, de Bruijn MT, et al: Accelerated perinecrotic outgrowth of colorectal liver metastases following radiofrequency ablation is a hypoxia-driven phenomenon. Ann Surg 249: 814-823, 2009.

26. St Hill CA: Interactions between endothelial selectins and cancer cells regulate metastasis. Front Biosci 16: 3233-3251, 2011.

27. Hofer EL, Labovsky V, La Russa V, et al: Mesenchymal stromal cells, colony-forming unit fibroblasts, from bone marrow of untreated advanced breast and lung cancer patients suppress fibroblast colony formation from healthy marrow. Stem Cells Dev 19: 359-370, 2010.

28. Oskowitz AZ, Lu J, Penfornis P, et al: Human multipotent stromal cells from bone marrow and microRNA: regulation of differentiation and leukemia inhibitory factor expression. Proc Natl Acad Sci USA 105: 18372-18377, 2008.

29. Whitney MJ, Lee A, Ylostalo J, Zeitouni S, Tucker A and Gregory CA: Leukemia inhibitory factor secretion is a predictor and indicator of early progenitor status in adult bone marrow stromal cells. Tissue Eng Part A 15: 33-44, 2009.

30. McAllister SS and Weinberg RA: Tumor-host interactions: a farreaching relationship. J Clin Oncol 28: 4022-4028, 2010.

31. Castano Z, Tracy K and McAllister SS: The tumor macroenvironment and systemic regulation of breast cancer progression. Int J Dev Biol 55: 889-897, 2011.

32. Hirbe AC, Morgan EA and Weilbaecher KN: The CXCR4/ SDF-1 chemokine axis: a potential therapeutic target for bone metastases? Curr Pharm Des 16: 1284-1290, 2010.

33. Meads MB, Hazlehurst LA and Dalton WS: The bone marrow microenvironment as a tumor sanctuary and contributor to drug resistance. Clin Cancer Res 14: 2519-2526, 2008.
34. Cronin PA, Wang JH and Redmond HP: Hypoxia increases the metastatic ability of breast cancer cells via upregulation of CXCR4. BMC Cancer 10: 225, 2010.

35. Teicher BA and Fricker SP: CXCL12 (SDF-1)/CXCR4 pathway in cancer. Clin Cancer Res 16: 2927-2931, 2010.

36. Kucia M, Jankowski K, Reca R, et al: CXCR4-SDF-1 signalling, locomotion, chemotaxis and adhesion. J Mol Histol 35: 233-245, 2004.

37. Petit I, Jin D and Rafii S: The SDF-1-CXCR4 signaling pathway: a molecular hub modulating neo-angiogenesis. Trends Immunol 28: 299-307, 2007.

38. Du R, Lu KV, Petritsch C, et al: HIF1alpha induces the recruitment of bone marrow-derived vascular modulatory cells to regulate tumor angiogenesis and invasion. Cancer Cell 13: 206-220, 2008

39. Sceneay J, Chow MT, Chen A, et al: Primary tumor hypoxia recruits $\mathrm{CD} 11 \mathrm{~b}^{+} / \mathrm{Ly} 6 \mathrm{Cmed} / \mathrm{Ly} 6 \mathrm{G}^{+}$immune suppressor cells and compromises NK cell cytotoxicity in the premetastatic niche. Cancer Res 72: 3906-3911, 2012.

40. Murphy ME: The HSP70 family and cancer. Carcinogenesis 34: 1181-1188, 2013

41. Teng LS, Jin KT, Han N and Cao J: Radiofrequency ablation, heat shock protein 70 and potential anti-tumor immunity in hepatic and pancreatic cancers: a minireview. Hepatobiliary Pancreat Dis Int 9: 361-365, 2010.

42. Ciocca DR and Calderwood SK: Heat shock proteins in cancer: diagnostic, prognostic, predictive, and treatment implications. Cell Stress Chaperones 10: 86-103, 2005.

43. Gibert B, Hadchity E, Czekalla A, et al: Inhibition of heat shock protein 27 (HspB1) tumorigenic functions by peptide aptamers. Oncogene 30: 3672-3681, 2011.

44. Wei L, Liu TT, Wang HH, et al: Hsp27 participates in the maintenance of breast cancer stem cells through regulation of epithelial-mesenchymal transition and nuclear factor-kappaB. Breast Cancer Res 13: R101, 2011.

45. Hsu HS, Lin JH, Huang WC, et al: Chemoresistance of lung cancer stemlike cells depends on activation of Hsp27. Cancer 117: 1516-1528, 2011.

46. Lin EY, Gouon-Evans V, Nguyen AV and Pollard JW: The macrophage growth factor CSF-1 in mammary gland development and tumor progression. J Mammary Gland Biol Neoplasia 7: 147-162, 2002.

47. Tsavaris N, Kosmas C, Vadiaka M, Kanelopoulos P and Boulamatsis D: Immune changes in patients with advanced breast cancer undergoing chemotherapy with taxanes. Br J Cancer 87: 21-27, 2002

48. Haen SP, Pereira PL, Salih HR, Rammensee HG and Gouttefangeas C: More than just tumor destruction: immunomodulation by thermal ablation of cancer. Clin Dev Immunol 2011: 160250, 2011.

49. Li J, Bouton-Verville H, Holmes LM, et al: Inhibition or promotion of tumor growth by granulocyte-macrophage colony stimulating factor derived from engineered tumor cells is dosedependent. Anticancer Res 24: 2717-2721, 2004.

50. Eubank TD, Roberts RD, Khan M, et al: Granulocyte macrophage colony-stimulating factor inhibits breast cancer growth and metastasis by invoking an anti-angiogenic program in tumoreducated macrophages. Cancer Res 69: 2133-2140, 2009. 